\title{
Synthesis and Design of Narrow-Band Micrwave Lossy Filter Based on Microstrip Technology
}

\author{
Akbar Kabirsalmani ${ }^{1}$, Majid Tayarani ${ }^{2}$, Farrokh Hojjat-Kashani ${ }^{2}$ \\ ${ }^{l}$ Department of Electrical Engineering, South Tehran Branch, Islamic Azad University, Tehran, Iran \\ ${ }^{2}$ Department of Electrical Engineering, Iran University of Science and Technology, Tehran, Iran
}

\begin{abstract}
Lossy synthesis techniques allow filters consisting resonators with finite quality factor $(Q)$ to be synthesized and designed while preserving prescribed in-band flatness. It is achieved at the expense of an overall constant additional insertion loss. As microstrip resonators generally suffer from low quality factor, using lossy synthesis techniques can be a good approach to design high performance narrow-band microstrip filters. In this paper, a lossy 3rd order Chebyshev filter with 5\% fractional bandwidth, based on microstrip technology is synthesized and designed. The filter is centered at $2.6 \mathrm{GHz}$ with the lossless return loss of $20 \mathrm{~dB}$ and a loss factor of $3.5 \mathrm{~dB}$. At first, the complex coupling matrix corresponding to the filter characteristic is synthesized. Then, it is transformed into a realizable form using trigonometric rotations. Afterwards, hyperbolic rotations are employed to evenly distribute the loss among resonators. The synthesized filter network is implemented using hairpin resonators with the $Q$ of 180. The simulation and optimization process is performed using a full-wave electromagnetic simulator (HFSS). Experimental evaluations illustrate that there is a reasonably good agreement between simulated and measured results.
\end{abstract}

Keywords : coupling matrix; lossy filters; matrix rotation; microstrip filters

\section{INTRODUCTION}

By the expansion of modern microwave systems, demands for high-performance and small size filters have been substantially increased during recent years. It is well known that the greatest hindrance to design these filters is the finite unloaded quality factor associated with resonators. Employing low-Q resonators in filters which are designed using conventional (lossless) methods causes a serious distortion in filter response [1], while high-Q resonators naturally tend to be heavy and massive or require expensive technologies. Therefore, designing high performance, and at the same time, small and low cost filters has been always a serious challenge to microwave engineers.

In order to include losses in the filter design procedure, lossy synthesis techniques have been introduced and several investigations have been conducted on them [2-7]. Lossy synthesis techniques enable filters with low-Q resonators to have ideal pass-band flatness at the expense of an additional absolute insertion loss which in most cases can be compensated by an amplifier without affecting the performance of the whole system [3].

Microstrip technology has extensive use in designing microwave filters due to its attractive features of low cost, light weight, capability of integration and repeatability. However, on the other hand, it suffers from relatively high conductor, dielectric and radiation losses. As a result, using conventional synthesis techniques, in which dissipative losses are not taken into consideration during the synthesis procedure, leads to degradation of narrowband microstrip filters response. Hence, utilizing lossy synthesis techniques to design of these filters can be a good approach to have a high-performance, small size and low-cost filter.

In this paper, a $3^{\text {rd }}$ order microstrip lossy filter with the center frequency of $2.6 \mathrm{GHz}$ and bandwidth of $130 \mathrm{MHz}$ is synthesized and designed. The in-band, lossless return loss is $20 \mathrm{~dB}$ and the scattering parameters are shifted down by $3.5 \mathrm{~dB}$. The design procedure is based on the coupling matrix synthesis technique. The method using which the complex coupling matrix element values are calculated is the one provided in [6]. According to this method, synthesizing the lossy $N+2$ coupling matrix is based on equating two expressions for Y-parameters which one of them is in terms of the residues of the $Y$-matrix (1), while the other is in terms of the circuit elements of the lossy transversal array (2).

$$
\begin{gathered}
{\left[Y_{N}\right]=j\left(\begin{array}{cc}
0 & K_{\infty} \\
K_{\infty} & 0
\end{array}\right)+\left(\begin{array}{cc}
g_{1} & 0 \\
0 & g_{2}
\end{array}\right)+\sum_{k=1}^{N} \frac{1}{\left(s-j \lambda_{k}\right)} \cdot\left(\begin{array}{cc}
r_{11 k} & r_{12 k} \\
r_{21 k} & r_{22 k}
\end{array}\right)} \\
{\left[Y_{N}\right]=j\left(\begin{array}{cc}
0 & J_{S L} \\
J_{S L} & 0
\end{array}\right)+\left(\begin{array}{cc}
G_{S} & 0 \\
0 & G_{L}
\end{array}\right)+\sum_{k=1}^{N} \frac{1}{\left(s C_{k}+j B_{k}+G_{k}\right)} \cdot\left(\begin{array}{cc}
J_{S K}^{2} & J_{S K} J_{L K} \\
J_{S K} J_{L K} & J_{L K}^{2}
\end{array}\right)}
\end{gathered}
$$


In (1), $K_{\infty}=0$, expect for the case in which the number of finite-position transmission zeros is exactly equal to the filter degree (fully canonical case). All the residues $\left(r_{i j k}\right)$ and eigenvalues $\left(\lambda_{k}\right)$ of the admittance matrix polynomial functions have complex values. Constant numbers $g_{1}$ and $g_{2}$ also remain from partial fraction expansion of $Y_{11}$ and $Y_{22}$, respectively. Fig. 1 illustrates the circuit model of the lossy transversal array with $N$ individual parallel connected lossy resonators, where $N$ is the filter degree. Here, each resonator consists of one capacitor $C_{k}$, one frequency invariant reactance $j B_{\mathrm{k}}$, and one resistance $G_{K}$. There are also two inverters, $J_{S K}$ and $J_{L K}$, on either side of each resonator connecting them to the source and load terminals.

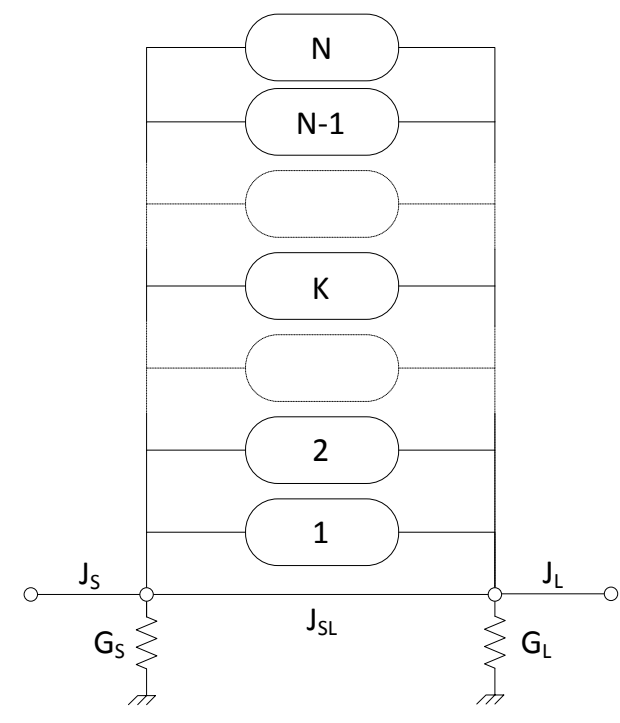

(a)

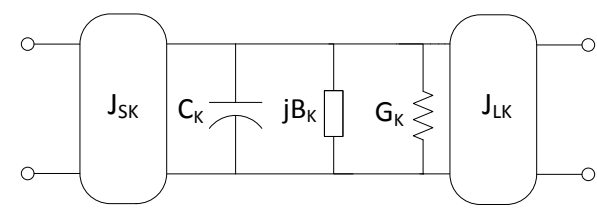

(b)

Fig. 1. Lossy canonical transversal array (a) $N$-Resonator transversal array including direct source-load coupling $\mathrm{J}_{S L}$ and (b) Equivalent circuit of the $k$ th lossy resonator in the transversal array.

\subsection{Coupling Matrix Calculation}

II.

FiLTER DeSign

Fig. 2 presents the normalized frequency response of a lossy third-order Chebychev filter, shifted down by $3.5 \mathrm{~dB}$ with the following scattering and admittance parameters:

$$
\begin{gathered}
S_{21}(p)=\frac{1.6625}{p^{3}+2.3434 p^{2}+3.4958 p+2.4875} \\
S_{11}(p)=\frac{0.6683 p^{3}+0.5013 p}{p^{3}+2.3434 p^{2}+3.4958 p+2.4875} \\
Y_{21}(p)=\frac{1.1946}{p^{3}+0.4659 p^{2}+2.1772 p+0.4945} \\
Y_{11}(p)=\frac{0.1988 p^{3}+1.218 p^{2}+0.695 p+1.2929}{p^{3}+0.4659 p^{2}+2.1772 p+0.4945}
\end{gathered}
$$

By partial fraction expansion of the admittance parameters and using (2), which makes a relation between Y-matrix and circuit elements of the transversal array (Fig. 1), the transversal coupling matrix is found and shown in Fig. 3. 


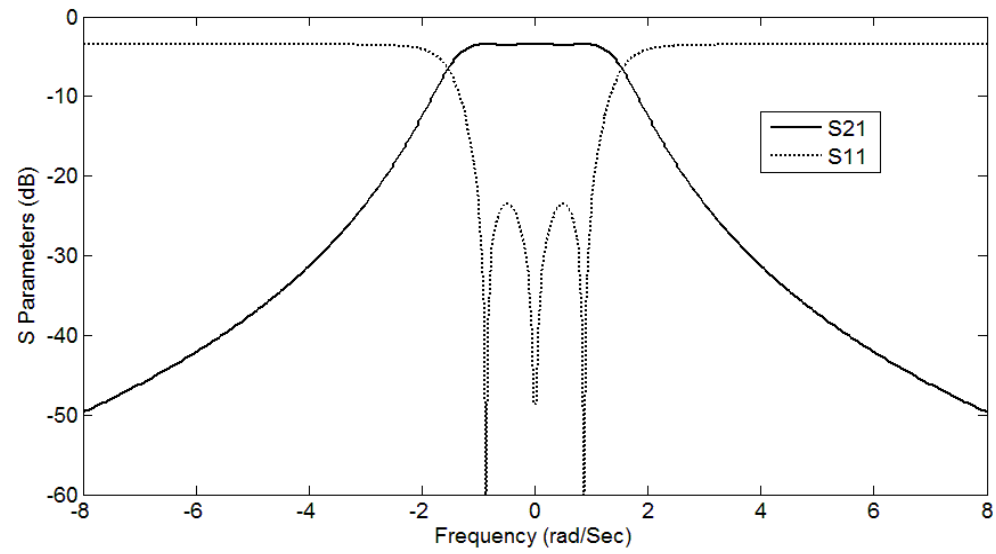

Fig. 2. Normalized frequency response of the Chebyshev three-pole lossy filter shifted down by $3.5 \mathrm{~dB}$

\begin{tabular}{l|ccccc|}
\multicolumn{1}{c}{} & $\mathrm{S}$ & 1 & 2 & 3 & $\mathrm{~L}$ \\
\cline { 2 - 6 } $\mathrm{S}$ & $-j 0.199$ & $0.531-j 0.021$ & -0.75 & $0.531+j 0.021$ & 0 \\
1 & $0.531-j 0.021$ & $1.452-j 0.116$ & 0 & 0 & $0.531-j 0.021$ \\
2 & -0.750 & 0 & $-j 0.233$ & 0 & 0.75 \\
3 & $0.531+j 0.021$ & 0 & 0 & $-1.452-j 0.116$ & $0.531+j 0.021$ \\
& & & & $0.531+j 0.021$ & $-j 0.199$ \\
$\mathrm{~L}$ & 0 & $0.531-j 0.021$ & 0.75 & 0.00
\end{tabular}

Fig. 3. Transversal coupling matrix of the Chebyshev three-pole lossy filter

Since the resultant coupling matrix is not physically realizable in its current form, a sequence of matrix transformations are required to obtain more suitable network topology having the same electrical response. One of the most convenient network configurations is achieved through folded coupling matrix. The whole process of transforming transversal coupling matrix into the folded form is fully described in [8]. The only difference here is that the rotation angles have complex values. In order to effectively distribute the loss throughout the network, hyperbolic rotation can be utilized [4]. The folded coupling matrix of the filter after adding two nonresonating nodes and distributing the loss evenly among the resonators is shown in Fig. 4. Here, the source and loud nodes have been scaled by a factor of 0.158 in order to remove the shunt resistors at source and load $\left(\mathrm{G}_{\mathrm{s}}\right.$ and $G_{L}$ in Fig. 1). The corresponding node diagram of the filter is depicted in Fig. 5. In this fig., red circles represent lossy resonators, blue ones point out non-resonating nodes, while S and L stand for source and load, respectively. Elements of the coupling matrix are also indicated by solid lines.

\begin{tabular}{c|ccccccc|}
\multicolumn{1}{c}{} & $\mathrm{S}$ & $\mathrm{NRN}_{\mathrm{S}}$ & 1 & 2 & 3 & $\mathrm{NRN}_{\mathrm{L}}$ & $\mathrm{L}$ \\
\cline { 2 - 8 } $\mathrm{S}$ & 0 & -0.158 & 0 & 0 & 0 & 0 & 0 \\
$\mathrm{NRN}_{\mathrm{S}}$ & -0.158 & $-j 0.005$ & -0.168 & $j 0.005$ & 0 & 0 & 0 \\
1 & 0 & -0.168 & $-j 0.172$ & 1.027 & $j 0.061$ & 0 & 0 \\
2 & 0 & $j 0.005$ & 1.027 & $-j 0.122$ & 1.027 & $j 0.005$ & 0 \\
3 & 0 & 0 & $j 0.061$ & 1.027 & $-j 0.172$ & -0.168 & 0 \\
$\mathrm{NRN}_{\mathrm{L}}$ & 0 & 0 & 0 & $j 0.005$ & -0.168 & $-j 0.005$ & -0.158 \\
$\mathrm{~L}$ & 0 & 0 & 0 & 0 & 0 & -0.158 & 0 \\
\hline
\end{tabular}

Fig. 4. Lossy coupling matrix of the filter in folded form 


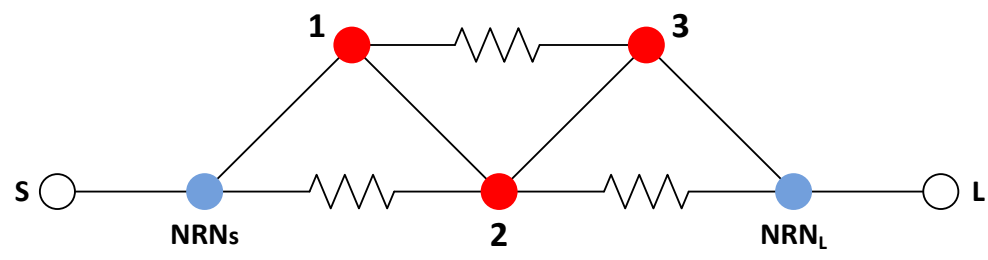

Fig. 5. Filter configuration after loss distribution

\subsection{Simulation and Measurement}

The synthesized filter network was simulated and optimized using a full-wave EM simulator Ansoft HFSS. The filter layout is exhibited in Fig. 6. Here, $\lambda / 2$ hairpin resonators with a $Q$ of 180 have been adopted to fulfil the desired loss factor. Transmission lines connecting non-resonating nodes to the first and last resonators have been folded to miniaturize the whole size of the filter. In Fig. 7 EM simulation response of the filter is shown.

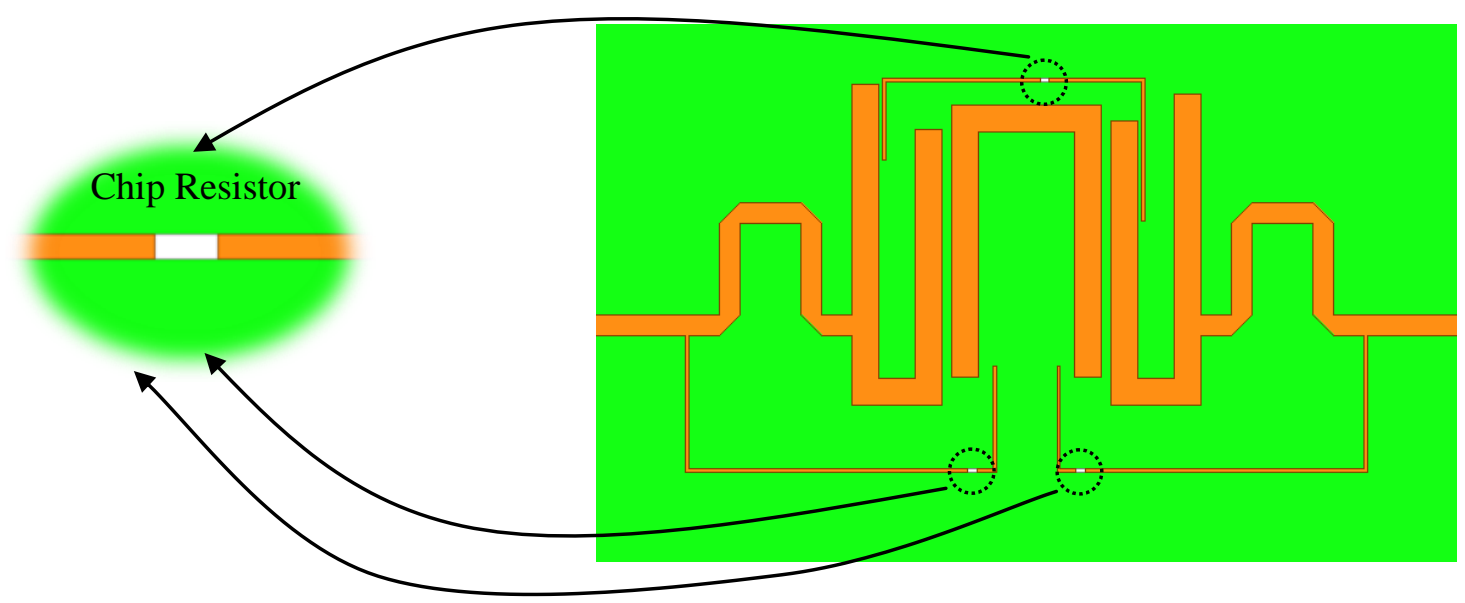

Fig. 6. HFSS model of the lossy filter

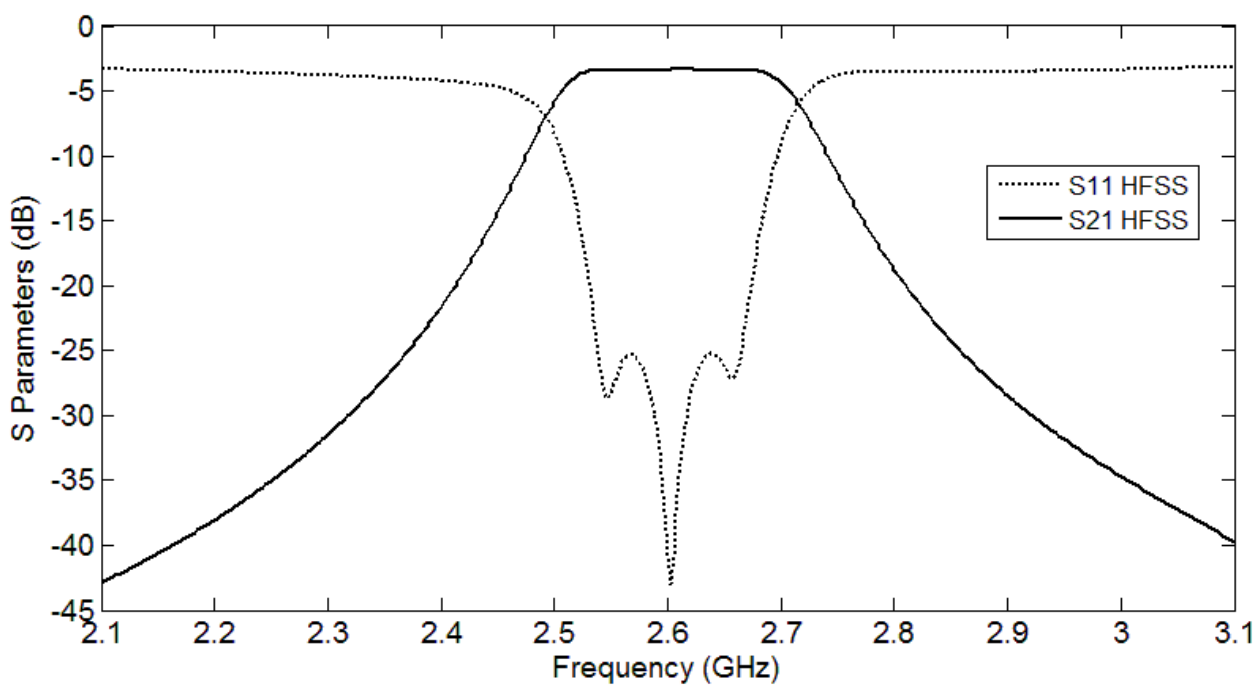

Fig. 7. EM simulation versus synthesis

The filter was fabricated on a 0.7874-mm-thick Rogers RO4003 with the dielectric constant of 2.38 and the loss tangent of $\tan \delta=0.0027$. Fig. 8 depicts the photograph of the fabricated filter. Resistive couplings were provided using 100-ohm transmission lines along with 0402 chip resistors. The comparison of the measured results and those obtained from EM simulations is presented in Fig. 9. The slight discrepancy between 
these two responses is largely due to parasitic behaviour of the resistors, their additional phase length, and also dielectric constant and manufacturing tolerance.

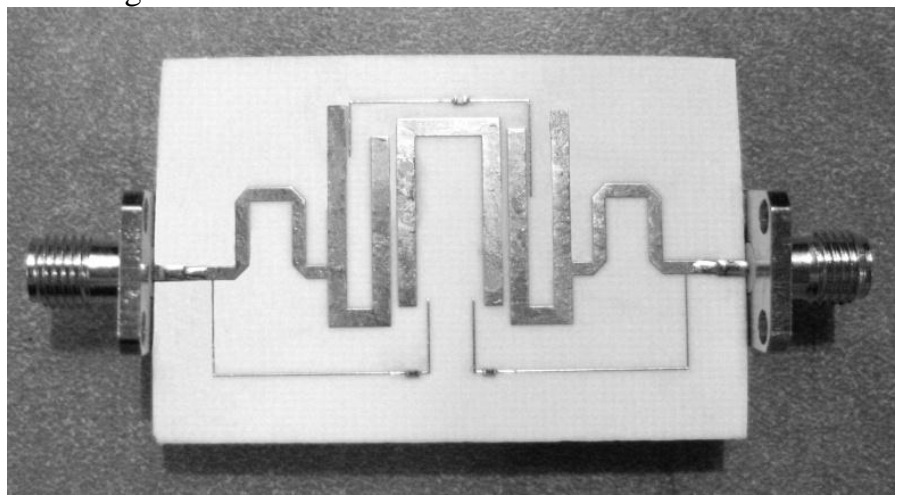

Fig. 8. Photograph of the fabricated filter

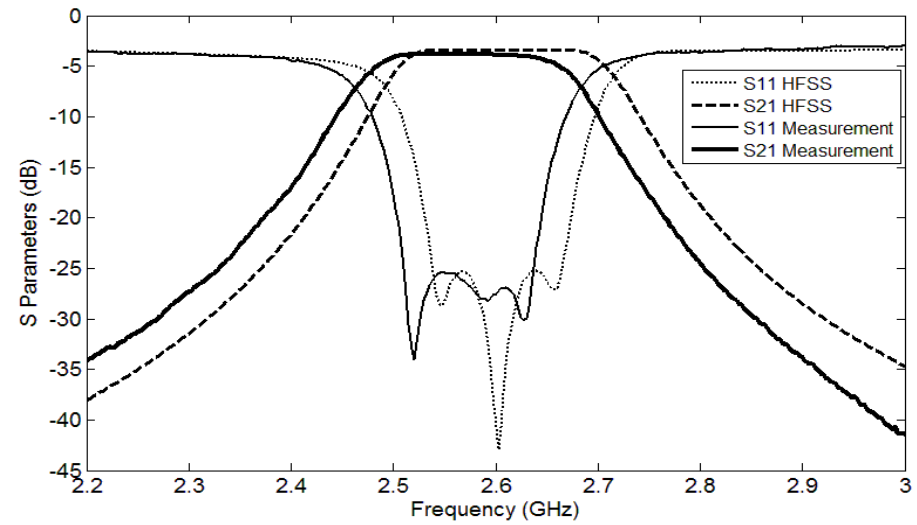

Fig. 9. Measurement versus EM simulation

\section{CONCLUSION}

Synthesis and design of a $3^{\text {rd }}$ order Chebyshev microstrip lossy filter was considered in this paper. Measured response of the manufactured filter confirms the design procedure. A relatively high performance filter with a flat in-band response was achieved using resonators with a $\mathrm{Q}$ of 180.

\section{REFERENCES}

[1] I.C. Hunter, Theory and design of microwave filters (The Institution of Engineering and Technology, 2001).

[2] B.S. Senior, I.C. Hunter, and J.D. Rhodes, Synthesis of lossy filters, Microwave Conference, 2002. 32nd European, 23-26 Sept. 2002, 2002; pp 1-4.

[3] A.C. Guyette, I.C. Hunter, and R.D. Pollard, A new class of selective filters using low-q components suitable for mmic implementation, Microwave Symposium Digest, 2004 IEEE MTT-S International, 6-11 June 2004, 2004; pp 1959-1962 Vol.1953.

[4] A.C. Guyette, I.C. Hunter, and R.D. Pollard, , The design of microwave bandpass filters using resonators with nonuniform Q. Microwave Theory and Techniques, IEEE Transactions on 2006, 54, 3914-3922.

[5] V. Miraftab and Y. Ming, Generalized lossy microwave filter coupling matrix synthesis and design using mixed technologies. Microwave Theory and Techniques, IEEE Transactions on 2008, 56, 3016-3027.

[6] V. Miraftab and Y. Ming, Advanced coupling matrix and admittance function synthesis techniques for dissipative microwave filters. Microwave Theory and Techniques, IEEE Transactions on 2009, 57, 2429-2438.

[7] M. Oldoni, G. Macchiarella, G.G. Gentili, and C. Ernst, A new approach to the synthesis of microwave lossy filters. Microwave Theory and Techniques, IEEE Transactions on 2010, 58, 1222-1229.

[8] R.J. Cameron, R. Mansour, and C.M. Kudsia, Microwave filters for communication systems. Wiley: 2007 ; p 804. 